\title{
Epidemiology of Cardiac Events During Prehospital Care in Mountain Rescues Conducted in Aragón
}

\author{
Carmen M. Martínez-Caballero, $\mathrm{MD}^{1}$; Eva Sierra Quintana, $\mathrm{MD}^{2}$ \\ ${ }^{1}$ Emergency Service of Castilla y León, Spain; ${ }^{2}$ Emergency Service of Aragón, Spain
}

\begin{abstract}
Introduction-Cardiac events are one of the leading causes of death in the Spanish population. Given the increase in the nontraumatic medical conditions found in mountain rescues, the objective of this study was to report on the heart conditions of patients rescued in the mountains of Aragón in the Spanish Pyrenees.

Methods-We conducted a retrospective observational study based on data collected from patients' medical histories for rescues undertaken in from 2010 to 2016 (at altitudes between $500 \mathrm{~m}$ [1640 ft] and $3404 \mathrm{~m}[11,168 \mathrm{ft}])$.

Results-Of the 2079 individuals rescued from 2010 to 2016, 34 (2\%) were diagnosed with heart conditions, accounting for $21 \%$ of all nontraumatic medical conditions. The data showed a statistically significant increase in the age of the rescued patients with heart conditions $(55 \pm 15 \mathrm{y})$ and those with acute coronary syndrome/sudden death $(60 \pm 8 \mathrm{y})$. Eighty-five percent of the rescued patients with heart conditions were men, $62 \%$ were rescued above $2000 \mathrm{~m}(6500 \mathrm{ft}), 42 \%$ had acute coronary syndrome (of whom 56\% had inferior infarction), and 35\% died suddenly.

Conclusions-Aragón mountain rescues show an increase in patient age in recent years. The most common medical case among rescued individuals with heart conditions was a hiker over the age of $50 \mathrm{y}$ with cardiovascular risk factors, inferior infarction, and occurrence at an altitude above $2000 \mathrm{~m}$. Based on our observations, appropriate training should be undertaken, especially by older hikers, who may also benefit from cardiac screening, and rescue vehicles/personnel and mountain huts should be equipped with semiautomatic external defibrillators.
\end{abstract}

Keywords: emergencies, acute coronary syndrome, sudden death, altitude

\section{Background}

The autonomous community of Aragón is located in northeastern Spain, bordering France and the middle of the Pyrenees. In 2015, 32\% of all deaths in Aragón were due to circulatory system disorders, the leading cause of death in Aragón.

The considerable variety of tourism options, especially in terms of physical fitness, makes this autonomous community a major tourist center for all age groups. Aragón's ski resorts offer winter sports, and the numerous natural habitats, such as the National Park of Ordesa and Monte Perdido, the natural parks of Posets-Maladeta, and Sierra de Guara and Sierra del

Corresponding author: Carmen M. Martínez-Caballero, MD, C/ Sayago 2 4B 47008 Valladolid, Spain; e-mail: cmmartinez@saludcastillayleon.es.

Submitted for publication March 2018.

Accepted for publication October 2018.
Moncayo, provide year-round activities. Many of Aragón's mountain peaks are above $3000 \mathrm{~m}$ (9800ft). These characteristics make Aragón's mountains a remote and rugged area, especially important for conducting mountain emergency rescue services.

The 061 Mountain Rescue Unit of Aragón, which is staffed by physicians and nurses, has been working jointly with rescue specialists and the Spanish Air National Guard since 2002. These collaborations have improved the medical care provided in the field and reduced the transfer time to definitive care facilities.

In recent years, there has been an increase in Aragón in the number of rescues and patients with nontraumatic medical conditions. From 2010 to 2016, 8\% of rescued patients had nontraumatic medical conditions, the most common of which were heart conditions $(21 \%)$, resulting in more than half of the deaths. ${ }^{1}$ 
Given the significance of cardiac events in the general population and the nonnegligible rate of heart conditions in rescued patients, we conducted a broader study of this type of rescued patient. Our ultimate objective is twofold: we aim to describe the main characteristics of rescues involving heart conditions and, based on these characteristics, we aim to provide recommendations for patients and healthcare staff to most effectively deal with the combination of heart disease and mountain activity.

\section{Methods}

An observational, descriptive, retrospective study was conducted of patients who presented with cardiac events and were treated by the 061 Mountain Rescue Unit of Aragón between July 2010 and December 2016. The study was authorized by the management of 061 of Aragón and by the clinical research ethics committee of Aragón.

The data were collected by members of the 061 Mountain Rescue Unit, who consulted the rescued patients' medical records and the database that stores information on rescues. The selection/inclusion criterion was presentation with a heart condition, defined by the presence of any of these symptoms: chest pain, palpitations, or syncope. Patients were diagnosed clinically through history and physical examination in the field and with electrocardiogram when possible. We excluded neurocardiogenic syncope with normal electrocardiogram and anxiety disorders. We analyzed the records of 34 patients and assigned each an identification number.

The following information was collected: demographic (age, sex, country of origin), clinical (type of condition, history, and risk factors), treatment (reperfusion therapy), and activity- and rescue-related (type of activity, year, season, altitude at which the patient was found, and transfer in an advanced life support [ALS] vehicle).

Data are presented as mean \pm SD (with range as appropriate). We performed a statistical analysis of the quantitative and qualitative variables using Student's t tests and $\chi^{2}$ tests, respectively. We analyzed the pattern of the number of rescues per year using a linear regression. The differences were considered statistically significant at $P<0.05$. All significant statistical tests reported in this article have power $=1-\beta$ greater than $95 \%$.

\section{Results}

During the study period (2010-2016), 2079 patients were rescued, $2 \%$ of whom had some type of heart condition. The number of rescued individuals with heart conditions was $5 \pm 3$ (range 1-10) per year. The data do not show any clear trend, except for a noticeable increase in 2016 (Figure 1).

The age of patients with heart conditions $(55 \pm 15$ [range 16-77] y) and of patients with acute coronary syndrome (ACS)/sudden death (60 \pm 8 [range 46-72] y) was significantly higher than the age of all rescued patients $(41 \pm 15$ [range 1-90] y) (independent samples $\mathrm{t}$ test, $P<0.001$ ) (Figures 2 and 3). There was a statistically significant difference in the age distribution, with heart conditions more common beyond $50 \mathrm{y}$ of age.

In terms of sex, $85 \%$ of the rescued patients were men. The age of the rescued men and women with heart conditions was 56 \pm 13 (range 16-77) y and 49 $\pm 21(28-72)$ y, respectively, with no statistically significant association.

Table 1 lists the conditions found according to the patients' sex and final disposition. There was a predominance of ACS (42\%) and sudden death caused by this

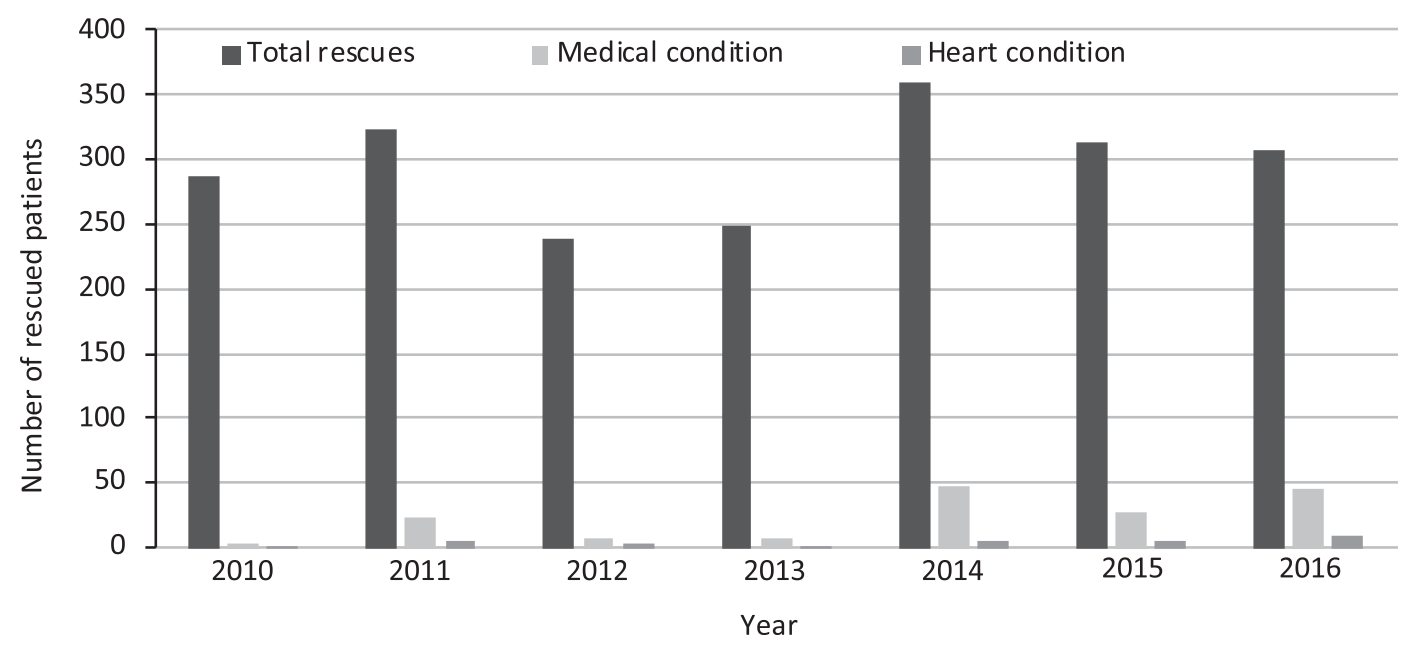

Figure 1. Evolution in the number of medical and heart disease rescues during the study. Mean \pm SD number of rescued individuals with heart conditions: $5 \pm 3$ (range $1-10$ ). 


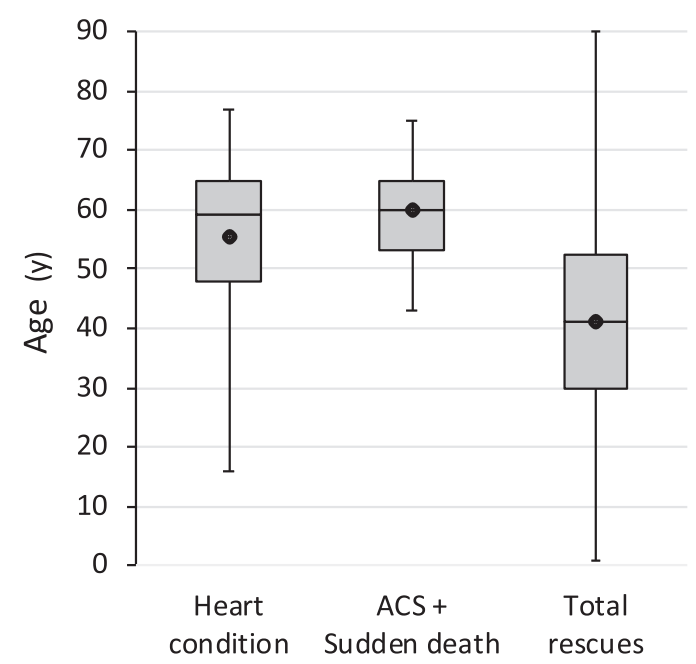

Figure 2. Box plots: From left to right, age of the rescued patients with heart disease, age of those with acute coronary syndrome (ACS) and sudden death, and age of the total of rescued patients (independent samples t test, $P<0.001)$. For each dataset, the point represents the mean. Horizontal lines correspond to the 3 quartiles, and the ends of the whiskers show the minimum and the maximum of the dataset.

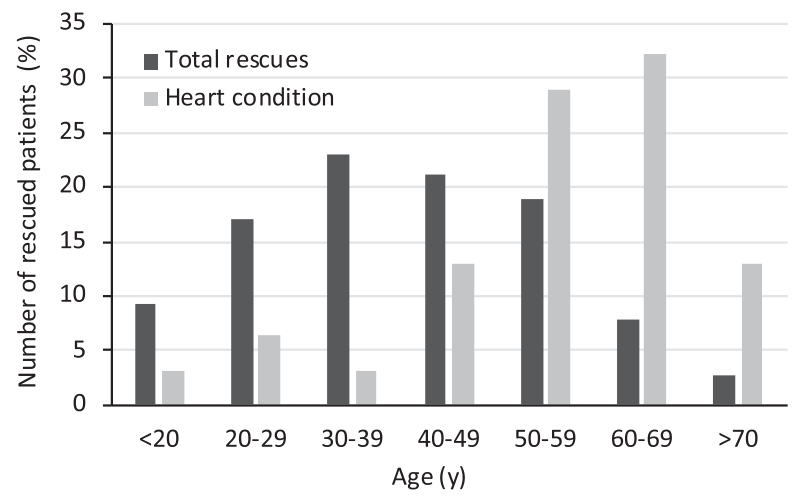

Figure 3. Distribution by age group of all rescues and of the patients with heart disease. Seventy-four percent of the patients with heart conditions were aged 50 years or older. The distributions are significantly different $\left(\chi^{2}\right.$ test, $\left.P<0.001\right)$.

syndrome $(35 \%)$ in men. In terms of the patient's final disposition, $56 \%$ of the patients were transferred to a hospital, $26 \%$ of whom were transferred by ALS vehicles, $12 \%$ by emergency medical units, and $15 \%$ by medical helicopters of the 112 emergency unit. Some $58 \%$ of the patients with ACS were transferred to hospital in an ALS transport.

Table 2 shows epidemiological characteristics regarding the most prevalent conditions in our patients. In terms of the patient's country of origin, Spain represented $65 \%$ of cases, and France represented 29\%; however, there was an equal number of Spanish and French deaths. In terms of patient sex, $80 \%$ of the women were French, and all were hikers. In terms of the altitude of event occurrence, $62 \%$ were rescued from above $2000 \mathrm{~m}$. The most common altitude range for ACS and death was 2000 to $2500 \mathrm{~m}$. There were more cases of sudden death at altitudes above $2000 \mathrm{~m}(64 \%)$. There were no reports of patients with ACS rescued above $2500 \mathrm{~m}$, although 1 of the patients was above this altitude engaging in sport activity before the coronary event. When relating altitude above $2000 \mathrm{~m}$ with the onset of ACS or sudden death, there were no statistically significant differences compared with the other conditions ( $\chi^{2}$ test $P=0.8$ ).

The patients who died in the field as a result of a cardiac event represented $32 \%$ of the rescued patients with heart conditions. The number of annual sudden deaths was $2 \pm 1$ (range 1-3), and the maximum number of deaths occurred both in 2014 and 2016.

The unit treated 12 patients with ACS, including 1 with cardiopulmonary arrest (CPA) who recovered after an acute myocardial infarction. The 12 patients corresponded to $35 \%$ of those rescued with heart conditions and were all men. The annual number of ACS cases was $2 \pm 2$ (range $0-5$ ) with $5(42 \%)$ of all studied cases of ACS occurring in 2016. This could well be related to the fact that a year before, the infarction code registry of Aragón was implemented. All of those patients were transferred to a hospital, 58\% in an ALS-equipped transport to optimize treatment and speed.

Clinical and treatment variables of the patients with ACS are described in the following. The most common risk factor of cardiac events in these patients was smoking; $50 \%$ of patients were smokers or had quit within the year before the event. Other risk factors were arterial hypertension (33\%) and dyslipidemia (25\%). One-third of these patients had a personal history of ischemic heart disease. The infarction was located in the inferior wall in more than half of the patients (56\%), and an anterior location was found in $33 \%$ of the patients.

In terms of the coronary revascularization therapy administered to these patients, percutaneous coronary intervention (PCI) was performed in at least $50 \%$ of cases, half of which were included in the infarction code registry of Aragón (the other PCIs were performed before the implementation of this protocol). In one case, fibrinolysis was performed followed by a rescue PCI.

\section{Discussion}

We have found no other article that analyzes mountain rescue cases with associated heart conditions in detail. Nonetheless, we have found studies that reported rates for rescues with associated heart conditions very similar to those found in our sample. In recent years, there has been a noticeable increase in nontraumatic medical 
Table 1. Cardiac or heart events found, patient's sex, and final outcome

\begin{tabular}{|c|c|c|c|c|c|c|c|}
\hline \multirow{2}{*}{$\begin{array}{l}\text { Cardiac or heart } \\
\text { events }(n=34)\end{array}$} & \multirow[t]{2}{*}{$n(\%)$} & \multicolumn{2}{|c|}{ Sex } & \multicolumn{4}{|c|}{ Outcome } \\
\hline & & $\begin{array}{c}\text { Male } \\
(85 \%) \\
n(\%)\end{array}$ & $\begin{array}{c}\text { Female } \\
(15 \%) \\
n(\%)\end{array}$ & $\begin{array}{c}\text { Hospital } \\
(56 \%) \\
n(\%)\end{array}$ & $\begin{array}{c}\text { Ambulatory } \\
\text { clinic }(6 \%) \\
n(\%)\end{array}$ & $\begin{array}{c}\text { No transport } \\
(6 \%) \\
n(\%)\end{array}$ & $\begin{array}{c}\text { Death } \\
(32 \%) \\
n(\%)\end{array}$ \\
\hline Angina/Chest pain & $2(6)$ & - & $2(40)$ & $2(10)$ & - & - & - \\
\hline Death after AMI & $11(32)$ & $10(35)$ & $1(20)$ & - & - & - & $11(100)$ \\
\hline Atrial flutter & $1(3)$ & $1(3)$ & - & - & $1(50)$ & - & - \\
\hline Pericarditis & $1(3)$ & $1(3)$ & - & $1(5)$ & - & - & - \\
\hline ACS & $12(35)$ & $12(42)$ & - & $12(64)$ & - & - & - \\
\hline Syncope + ECG ab. & $5(15)$ & 4 (14) & $1(20)$ & $3(16)$ & $1(50)$ & $1(50)$ & - \\
\hline Tachycardia & $1(3)$ & $1(3)$ & - & $1(5)$ & - & - & - \\
\hline PSVT & $1(3)$ & - & $1(20)$ & - & - & $1(50)$ & - \\
\hline
\end{tabular}

AMI, acute myocardial infarction; ACS, acute coronary syndrome; ECG ab., electrocardiogram abnormalities; PSVT, paroxysmal supraventricular tachycardia.

Table 2. Epidemiological characteristics

\begin{tabular}{|c|c|c|c|}
\hline Variable & $\begin{array}{c}\text { Total cardiac } \\
\text { or heart events } \\
(n=34) n(\%)\end{array}$ & $\begin{array}{c}A C S \\
(n=12) \\
n(\%)\end{array}$ & $\begin{array}{c}\text { Sudden death } \\
(n=11) \\
n(\%)\end{array}$ \\
\hline \multicolumn{4}{|l|}{ Sex } \\
\hline Male & $29(85)$ & $12(100)$ & $10(91)$ \\
\hline Female & $5(15)$ & - & $1(9)$ \\
\hline \multicolumn{4}{|l|}{ Season } \\
\hline Winter & $1(3)$ & $1(8)$ & - \\
\hline Spring & $4(12)$ & $1(8)$ & $3(27)$ \\
\hline Summer & $24(70)$ & $8(67)$ & $5(46)$ \\
\hline Autumn & $5(15)$ & $2(17)$ & $3(27)$ \\
\hline \multicolumn{4}{|l|}{ Sports activity } \\
\hline Canyoning & $3(9)$ & - & $1(9)$ \\
\hline Hunting & $1(3)$ & - & $1(9)$ \\
\hline Climbing & $1(3)$ & - & - \\
\hline Caving & $1(3)$ & $1(8)$ & $1(9)$ \\
\hline $\begin{array}{l}\text { Cross-country } \\
\text { skiing }\end{array}$ & $1(3)$ & $1(8)$ & - \\
\hline Hiking & 27 (79) & $10(84)$ & $8(73)$ \\
\hline \multicolumn{4}{|l|}{ Altitude, m } \\
\hline $500-1000$ & $7(20)$ & - & $3(27)$ \\
\hline $1000-2000$ & $6(18)$ & $5(42)$ & $1(9)$ \\
\hline $2000-3000$ & $19(56)$ & $7(58)$ & $6(55)$ \\
\hline Over 3000 & $2(6)$ & - & $1(9)$ \\
\hline
\end{tabular}

ACS, acute coronary syndrome.

conditions in patients rescued in Aragón, from 8\% to $12 \%$ in the past 3 y (2014-2016). Heart disease was the second leading nontraumatic medical condition after environmental medical conditions, representing $21 \%$ of patients rescued from 2010 to $2016 .{ }^{1}$ In New Zealand, medical conditions represented $12 \%$ of rescue operations conducted from May 2010 to December 2011, and cardiovascular events were reported as the most common. ${ }^{2}$ The rates were similar to those found in US studies in which the predominant cause of death within nontraumatic events was cardiovascular disorder. ${ }^{3}$ In the United States, similar rates of heart problems and sudden death were reported for rescues in Denali (approximately $17 \%$ ), and cardiac arrest after AMI was considered the largest single cause of death observed in Baxter State Park. ${ }^{4,5}$

The progressive increase in the number of rescues and patients with heart conditions is reflected globally in North America, Europe, and Asia. ${ }^{6}$ This trend suggests there will be a greater concern for increased surveillance and for the implementation of preventive measures to keep up with the growth of recreational sports practice in modern society.

At the start of the 1990s, the mean age of those rescued was $37 \mathrm{y}$. By 2012, that age had increased to $45 \mathrm{y}$, and by 2016 (ie, our study's period), the age had reached $55 \mathrm{y} .{ }^{6}$ The age increases to $59 \mathrm{y}$ if we include ACS and sudden death, with a clear majority of the patients being men. ${ }^{7}$ The longer life expectancy and the greater availability of leisure time could explain the increase in the mean age of those rescued in recent decades. Atherosclerosis gradually progresses with age, and therefore age is a predominant risk factor in coronary artery disease. ${ }^{8}$

Hiking, the most popular physical fitness activity, ${ }^{1,2,4,9,10}$ has benefits associated with healthy lifestyles and increased longevity. However, a lack of physical fitness, dehydration, excessive exercise intensity, and exposure to altitude can trigger adverse cardiac events in individuals with risk factors. ${ }^{11}$

Although $62 \%$ of the patients who had a heart condition when rescued were found at an altitude higher than $2000 \mathrm{~m}$ (increasing to $64 \%$ for the patients who died), 
there was no statistically significant association. However, there are studies that have related myocardial ischemia with altitude. Reports from Mont Blanc have indicated between 5 and 10 cases of myocardial ischemia per year, probably because of the lack of high altitude adaptation and the rapid performance of intense activity. $^{7}$

Hypoxic conditions stimulate the sympathetic system, increasing the heart rate, cardiac contractility, and platelet aggregation. The onset of respiratory alkalosis can trigger arrhythmias, and the increased cardiac demand can cause myocardial ischemia. ${ }^{8,12}$ This situation, coupled with environmental conditions, intense exercise, dehydration, diet change, and emotional stress from dangerous conditions, can precipitate myocardial dysfunction.

A third of the patients in our sample had a history of ischemic heart disease, the symptoms of which can be exacerbated by altitude. Heart conditions are more common among older untrained men during the first 5 days of high altitude exposure. An optimal level of training is therefore recommended, along with appropriate acclimatization (at least $5 \mathrm{~d}$ ), optimal medication control (especially statins and aspirin), and a stress test to assess the body's capacity for exercise and the potential onset of ischemia. ${ }^{7}$ With this in mind, most older patients with heart disease can tolerate exposure to high altitudes safely with a minimum increase in risk. ${ }^{8}$

Such cases will inexorably occur, and when they do, they will have to be managed in remote locations far from advanced medical services. The expertise of first responders and the rapid application of emergency services are vital to prevent disastrous results.

In urban settings, survival rates for CPA outside hospitals are low, with a 70 to $98 \%$ failure rate for resuscitation attempts. ${ }^{13}$ The continuity of the survival chain is essential for favorable results ${ }^{14,15}$ and represents a major challenge for CPAs in remote locations.

Patients in mountaineering environments are expected to have poorer survival rates than those in urban areas because of the remoteness of their rescue location, whose characteristics (access difficulty, terrain, restricted means, and personnel) most often delay the start of treatment and the subsequent transfer to hospital. However, this finding is balanced somewhat by the fact that patients who engage in these types of activities are generally healthier than those in urban studies of CPA. ${ }^{16}$

Studies have shown that training and the systematic use of semiautomatic external defibrillators (SAEDs) help reduce mortality. ${ }^{17}$ In 2006, the International Commission for Alpine Rescue recommended the use of SAEDs, stating that approximately $50 \%$ of deaths during mountain hiking were from sudden death by cardiac arrest.
Rescue teams are therefore advised to carry an SAED to improve the results of emergency care in these cases. ${ }^{18,19}$ Supplying SAEDs to mountain huts and youth hostels in the mountains of Aragón is essential, as is promoting the training of mountain rangers and mountaineers in the use of SAEDs and first aid techniques. These initiatives increase survival chances by minimizing the time to the first defibrillation. The only patient with CPA in our study who was successfully resuscitated benefited from cardiopulmonary resuscitation carried out by onscene bystanders and the use of an SAED located in a nearby mountain hut.

Treatment for patients with ACS has been optimized through Aragón's current infarction code protocols, ${ }^{20}$ shortening the time to diagnosis, increasing survival, and minimizing the time to revascularization therapy (through fibrinolysis and/or PCI). These protocols require the support of ALS vehicles for fibrinolysis and/ or transfer to an equipped hospital.

\section{STUDY LIMITATIONS}

Despite covering $7 \mathrm{y}$, the study's small sample size resulting from the low incidence of submitted cases precludes us from establishing unequivocal conclusions. The fact that there are no studies on mountain rescues with details on the heart condition of rescued patients prevents us from comparing the results.

The epidemiological characteristics of rescued patients may justify further research and observational work, both to efficiently guide practitioners toward the patient type that could be encountered and to establish effective preventive mechanisms.

\section{Conclusions}

Cardiac disease is an important cause of morbidity and mortality in the Pyrenees among those requiring rescue. Age is directly associated with a greater risk of presenting with adverse cardiac events. We are faced with an increasingly longer-lived population with more leisure time and an enjoyment of outdoor activities, with all the risks that this implies.

Based on our observations, the most common medical case among rescued individuals with heart conditions was a summer hiker over the age of $50 \mathrm{y}$ with a history of cardiovascular risk factors (smoking, hypertension, or history of ischemia) who experienced an infarction (most frequently an inferior wall infarction) and occurrence at an altitude above $2000 \mathrm{~m}$. This type of patient will probably need to be transferred to a hospital and could benefit from an SAED-equipped rescue team.

In terms of prevention, progressive and healthy training is therefore recommended. For the same reasons, a 
stress test to unmask silent ischemic heart disease should be considered before the performance of any remote activity that requires strenuous exercise, at least by the population with cardiovascular risk factors.

Acknowledgments: The authors thank the 061 Mountain Rescue Unit of Aragón for sharing their data. They are very grateful to L. Izquierdo for his patience and guidance during the study. They thank the editor and the anonymous reviewers whose comments helped us to improve this article.

Author Contributions: Study concept and design (CM, ES); data acquisition (ES); analyzing data (CM, ES); drafted and critically reviewed the manuscript $(\mathrm{CM})$; approved final manuscript (CM, ES).

Funding/Material Support: None.

Disclosures: None

\section{References}

1. Sierra Quintana E, Martínez Caballero CM, Batista Pardo SA, Abella Barraca S, De La Vieja Soriano M. Patología médica no traumática en pacientes rescatados en montaña. Emergencias. 2017;29(5):339-42.

2. Visser JT, Campbell AFR. New Zealand land search and rescue operations: an analysis of medical and traumatic conditions. Wilderness Environ Med. 2014;25(4):401-8.

3. Boore SM, Bock D. Ten years of search and rescue in Yosemite national park: examining the past for future prevention. Wilderness Environ Med. 2013;24(1):2-7.

4. McIntosh SE, Brillhart A, Dow J, Grissom CK. Search and rescue activity on Denali, 1990 to 2008. Wilderness Environ Med. 2010;21(2):103-8.

5. Welter CR, Sholl JM, Strout TD, Woodard B. Epidemiology of search and rescue in Baxter State Park: Dangers of descent and fatigue. Wilderness Environ Med. 2015;26(4):549-54.

6. Ciesa M, Grigolato S, Cavalli R. Retrospective study on search and rescue operations in two prealps areas of Italy. Wilderness Environ Med. 2015;26(2):150-8.

7. Marsigny B, Lecoq-Jammes F, Cauchy E. Medical mountain rescue in the Mont-Blanc massif. Wilderness Environ Med. 1999;10(3):152-6.

8. Levine BD. Going high with heart disease: the effect of highaltitude exposure in older individuals and patients with coronary artery disease. High Alt Med Biol. 2015;16(2):89-96.

9. Martínez Caballero C, López I, Sierra Quintana E, Caso E. Montaña en solitario: Estudio clínico-epidemiológico de los rescates realizados por el grupo de rescate especial de intervención en montaña de la Guardia Civil (GREIM) durante el periodo 2010-2014 en la Comarca del Sobrarbe (Huesca-España). Abstract book from the International Workshop of Mountain Medicine. CIMA2015, March 26-28, 2015. Zaragoza, Spain: University of Zaragoza; 2015:159-62. ISBN-13: 978-84-606-6963-0.

10. Heggie TW. Search and rescue in Alaska's national parks. Travel Med Infect Dis. 2008;6(6):355-61.

11. Burtscher M, Ponchia A. The risk of cardiovascular events during leisure time activities at altitude. Prog Cardiovaso Dis. 2010;52(6):507-11.

12. Rimoldi SF, Sartori C, Seiler C, Delacrétaz E, Mattle HP. High-altitude exposure in patients with cardiovascular disease: risk assessment and practical recommendations. Prog Cardiovasc Dis. 2010;52(6):512-24.

13. Burtscher M. Risk of cardiovascular events during mountain activities. Adv Exp Med Biol. 2007;618:1-11.

14. Yanagawa Y, Omori K, Takuchi I, Jitsuiki K, Yoshizawa $\mathrm{T}$, Ishikawa $\mathrm{K}$, et al. Cardiac arrest at high elevation with a favorable outcome. Am J Emerg Med. 2017;35(4):661. e5-7.

15. Ströhle M, Paal P, Strapazzon G, Avancini G, Procter E, Brugger H. Defibrillation in rural areas. Am J Emerg Med. 2014;32(11):1408-12

16. Paal P, Milani M, Brown D, Boyd J, Ellerton J. Termination of cardiopulmonary resuscitation in mountain rescue. High Alt Med Biol. 2012;13(3):200-8.

17. Hallstrom A, Ornato J. Public-access defibrillation and survival after out-of-hospital cardiac arrest. $N$ Engl $\mathrm{J} \mathrm{Med.}$ 2004;351:637-46.

18. Elsensohn F, Agazzi G, Syme D, Swangard M, Facchetti G, Brugger $H$. The use of automated external defibrillators and public access defibrillators in the mountains: official guidelines of the International Commission for Mountain Emergency Medicine ICAR-MEDCOM. Wilderness Environ Med. 2006;17(1):64-6.

19. Barcella L, Agazzi G, Malgrati D, et al. First aid and public access defibrillation in mountain huts: the mountain huts initiative of the bergamo section of the Club Alpino Italiano. Wilderness Environ Med. 2010;21(4):379-81.

20. Lukin Otanovic A. Código infarto Aragón. III Workshop Semergen-Aragón: Zaragoza, Spain, November 20-21, 2015 . 